\title{
Immunotherapeutic treatments for spinal and peripheral nerve tumors: a primer
}

\author{
Jean-Paul Bryant, MS, Victor M. Lu, MD, PhD, Vaidya Govindarajan, BS, \\ Roberto J. Perez-Roman, MD, and Allan D. Levi, MD, PhD \\ Department of Neurological Surgery, University of Miami Miller School of Medicine, Miami, Florida
}

OBJECTIVE Spinal and peripheral nerve tumors are a heterogeneous group of neoplasms that can be associated with significant morbidity and mortality despite the current standard of care. Immunotherapy is an emerging therapeutic option to improve the prognoses of these tumors. Therefore, the authors sought to present an updated and unifying review on the use of immunotherapy in treating tumors of the spinal cord and peripheral nerves, including a discussion on mechanism of action, drug delivery, current treatment techniques, and preclinical and clinical studies.

METHODS Current data in the literature regarding immunotherapy were collated and summarized. Targeted tumors included primary and secondary spinal tumors, as well as peripheral nerve tumors.

RESULTS Four primary modalities of immunotherapy (CAR T cell, monoclonal antibody, viral, and cytokine) have been reported to target spine and peripheral nerve tumors. Of the primary spinal tumors, spinal cord astrocytomas had the most preclinical evidence supporting immunotherapy success with CAR T-cell therapy targeting the H3K27M mutation, whereas spinal schwannomas and ependymomas had the most evidence reported for monoclonal antibody therapy preclinically. Of the secondary spinal tumors, primary CNS lymphomas demonstrated some clinical response to immunotherapy, whereas multiple myeloma and bone tumor experiences with immunotherapy were largely limited to concept only. Within peripheral nerve tumors, the use of immunotherapy to treat neurofibromas in the setting of syndromes has been suggested in theory, and possible immunotherapeutic targets have been identified in malignant peripheral nerve tumors. To date, there have been 2 clinical trials involving spine tumors and 2 clinical trials involving peripheral nerve tumors that have reported results, all of which are promising but require validation.

CONCLUSIONS Immunotherapy to treat spinal and peripheral nerve tumors has become an emerging area of research and interest. A large amount of preclinical data supporting the translation of this therapy into practice, aimed at ameliorating the poor prognoses of specific tumors, have been reported. Future clinical studies for translation will focus on the optimal therapy type and administration route to best target these tumors, which often preclude total surgical resection given their proximity to the neural and vascular elements of the spine.

https://thejns.org/doi/abs/10.3171/2021.11.FOCUS21590

KEYWORDS immunotherapy; spine tumors; peripheral nerve tumors; CAR T cell; vaccines

$\mathrm{S}$ PINAL and peripheral nerve tumors encompass a diverse group of neoplasms with the potential to cause significant neurological morbidity and even mortality when not appropriately treated. Traditionally, treatment of these tumors has included surgery, radiation therapy, and chemotherapy. Resection of benign spinal tumors is often curative, while high-grade lesions in the spine can carry an exceedingly poor prognosis., ${ }^{1,2}$ Similarly, peripheral nerve tumors are characterized by wide variability in tumor behavior and clinical prognoses. ${ }^{3}$ While advancements in resection, chemotherapy, and radiation therapy have improved clinical outcomes in patients with spinal and peripheral nerve tumors, these high-grade neoplasms are nonetheless associated with exceedingly low 5-year survival rates. ${ }^{1,2}$ Furthermore, surgical excision is often difficult, given the tendency of these tumors to infiltrate along the neural elements, which limits the extent of resection. This creates a need for alternative treatment options that may serve as an adjunct to the treatment armamentarium. One such modality that has garnered substantial recent attention in the neuro-oncology literature is the use of immunotherapeutics. ${ }^{4-10}$

ABBREVIATIONS BCMA = B-cell maturation antigen; CAR = chimeric antigen receptor; $\mathrm{DMG}=$ diffuse midline glioma; EGFR = epidermal growth factor receptor; IFN- $\alpha=$ interferon- $\alpha ; \mathrm{IL}=$ interleukin; MPNST = malignant peripheral nerve sheath tumor; NF1 = neurofibromatosis type 1; NF2 = neurofibromatosis type 2; PCNSL = primary CNS lymphoma; PD-1 = programmed cell death protein 1; SCA = spinal cord astrocytoma; VEGF = vascular endothelial growth factor.

SUBMITTED October 1, 2021. ACCEPTED November 17, 2021.

INCLUDE WHEN CITING DOI: 10.3171/2021.11.FOCUS21590. 


\section{Immunotherapy for Tumors}

There are several notable varieties of immunotherapy currently under investigation in the setting of CNS tumors. Therapy using chimeric antigen receptor (CAR) T cells, which are engineered $\mathrm{T}$ cells that target antigens found on tumor cell surfaces, are of great interest. ${ }^{1-4}$ They provide a significant advantage in tumor specificity as their antigenrecognition domains are selective for overexpressed or upregulated surface tumor antigens. Similarly, another strategy includes monoclonal antibodies, which are an older version of a similar concept, as they are synthetic proteins that either directly interrupt cellular processes or opsonize tumor cells by binding to surface antigens. Less-studied viral and cytokine therapies are less-selective forms of immunotherapy that leverage their abilities to induce general immune responses against tumor cells. ${ }^{11}$

While immunotherapy for malignant brain tumors such as glioblastoma has been well described, less is known regarding the use of immunotherapeutics in spinal and peripheral nerve tumors. There are multiple avenues to access the spinal cord and peripheral nerves for immunotherapy delivery, including intravenous delivery, ${ }^{4,12}$ intratumoral delivery, ${ }^{13-16}$ and intrathecal delivery. ${ }^{17,18}$ Herein, we present an updated and unifying review on the use of immunotherapy in treating tumors of the spinal cord and peripheral nerves, including a discussion on mechanism of action, drug delivery, current treatment techniques, and preclinical and clinical studies (Fig. 1).

\section{Primary Spinal Cord Tumors}

Primary tumors of the spinal cord are relatively rare and occur less often than metastatic lesions. Primary intramedullary spinal cord tumors are rare entities only affecting approximately $5 \%$ of patients with primary CNS lesions. ${ }^{19}$ The most commonly occurring intramedullary tumors include ependymomas and astrocytomas, which typically portend to poor prognoses. ${ }^{20}$ Additionally, spinal tumors may arise in the extramedullary space, such as melanotic spinal schwannomas, which have been studied as targets of immunotherapeutic agents.

\section{Spinal Cord Astrocytoma}

Spinal cord astrocytomas (SCAs) constitute approximately $6 \%$ to $8 \%$ of all primary intradural spinal cord tumors. ${ }^{1}$ Nearly $75 \%$ of these primary SCAs are low grade, while the remaining present as high-grade lesions. ${ }^{2}$ Safe resection is often curative in low-grade lesions; however, the propensity of these tumors to infiltrate surrounding critical vasculature and spinal cord elements limits the extent of resection. Therefore, surgical intervention is not always associated with significant increases in overall survival, with some studies reporting mortality rates as high as $70 \%$ within 6 months after diagnosis and resection. ${ }^{1}$

While these preclinical studies show promise, there are few clinical studies or case reports examining the use of immunotherapy to treat SCAs. CAR T-cell therapy was first adopted in CNS tumors to treat intracranial glioblastoma. ${ }^{4,14,21-25}$ Three target-specific antigens, which include epidermal growth factor receptor (EGFR) vIII, ephrin type-A receptor 2 (EphA2), and interleukin (IL) 13R $\alpha 2$,

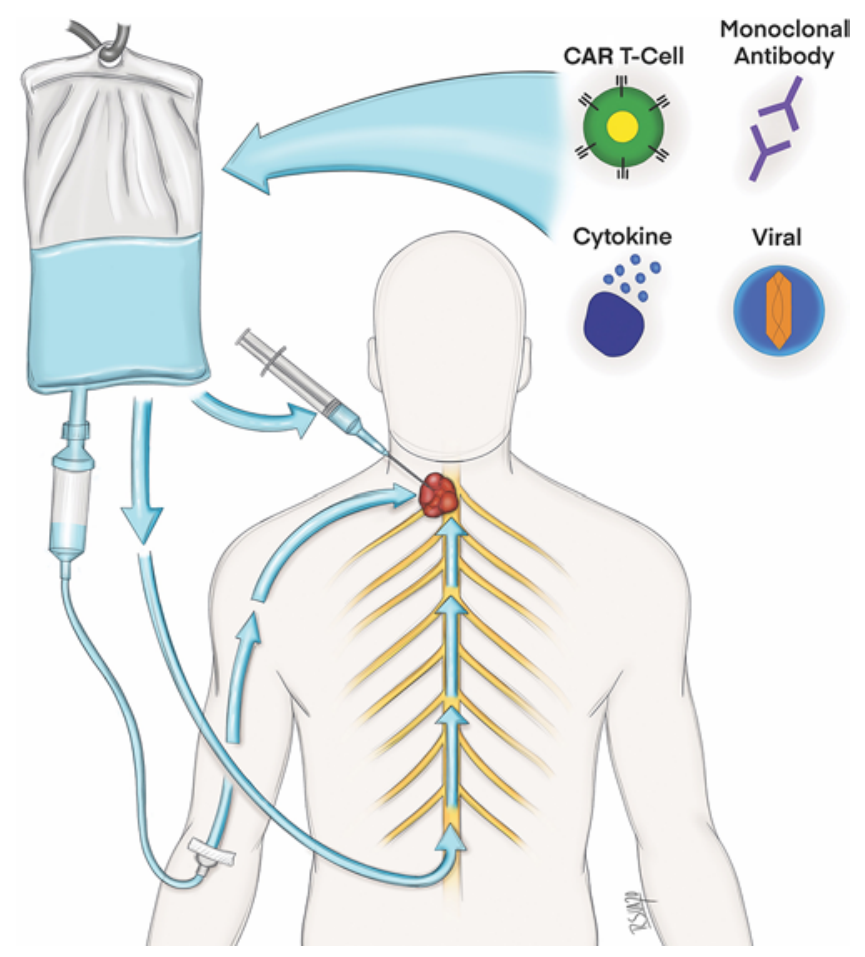

FIG. 1. Illustrative diagram of summarized immunotherapeutic agents and possible administration routes (intravenous, intrathecal, and intratumoral) to target spine and peripheral nerve tumors. Copyright Roberto Suazo. Published with permission.

have been previously identified in adult glioblastoma. ${ }^{1,12}$, ${ }^{26,27}$ While immunotherapeutic studies have shown potential for these targets, they occur primarily intracranially, rendering these epitopes not suitable for SCA-targeted therapy. ${ }^{3,28-30}$ However, a recent study by Mount et al. ${ }^{31}$ reported that $\mathrm{H} 3 \mathrm{~K} 27 \mathrm{M}$ mutations may serve as an appropriate target for treating SCAs with immunotherapeutic techniques. ${ }^{31}$ In this study, disialoganglioside GD2 was characterized as a specific tumor cell marker that was upregulated in diffuse midline glioma (DMG) but absent on normal cellular surfaces. The investigators then developed an H3K27M+DMG xenograft mouse model in which the graft was extracted from 5 patients with thalamic or spinal cord high-grade gliomas. Experiments were conducted to test the efficacy of the antitumor response generated by anti-GD2 CAR T-cell therapy. Anti-GD2 CAR T cells produced cytotoxic cytokines that selectively killed tumor cells in these mouse models. Moreover, transplanted tumors demonstrated a significant reduction in size compared with controls in 2 patient-derived xenograft models, highlighting the efficacy of the targeted immunotherapy. ${ }^{31}$ While this study provides excellent insight into the use of CAR T cells in treating SCAs, it is not without limitations. First, SCA tumor cells were not engrafted directly into the spinal cord but rather into the medulla to avoid paralysis. SCAs were still detected diffusely in the CNS via bioluminescence and demonstrated significant growth within the spinal cord. Next, loss of GD2 antigen expression did occur, which resulted in elimination of cytokine production from anti-GD2 CAR T cells compared with 
untreated or treated DMG cells. Despite these limitations, this study was novel in demonstrating the cytotoxic potential of these CAR T cells for SCA therapy.

In addition to CAR T-cell therapy, the use of therapeutic vaccines has garnered considerable attention in the immunotherapeutic literature..$^{32-34}$ While the subject is well studied, the therapeutic efficacy of this modality remains unclear. However, the specificity of the H3K27M mutation for SCAs provides a selective target for peptide or neoantigen vaccines, which was evaluated by Ochs et al. and Chheda et al. in preclinical investigations. ${ }^{35,36} \mathrm{In}$ their work, Ochs et al. found that a peptide vaccine against K27M-mutant histone 3 was able to induce specific cytotoxic T-cell- and T-helper-1-cell-mediated responses in a mouse model. ${ }^{36}$ Chheda et al. observed that Toll cell receptor-transduced $\mathrm{T}$ cells selectively killed $\mathrm{H} 3 \mathrm{~K} 27 \mathrm{M}$ glioma cells in an antigen-specific manner, thus supporting the development of a T-cell-based therapy of this neoantigen in high-grade gliomas. ${ }^{35}$

\section{Schwannomas}

True spontaneous spinal schwannomas are very rare, and commonly occur in the setting of trauma following Schwann cell proliferation after spinal cord injury (this is known as schwannomatosis). ${ }^{37}$ All other spinal schwannomas arise for peripheral nerve rootlets, most commonly, the dorsal roots..$^{38}$ One additional type of schwannoma in this setting is melanotic schwannoma, a nerve sheath tumor with a uniform composition of variably melaninproducing Schwann cells and metastatic potential. These schwannomas can occasionally extend into the spinal cord as well.

The use of immunotherapy in schwannomas is limited to case series. Solomou et al. reported the use of multiple immunotherapeutics in the management of a patient with advanced melanotic spinal schwannoma. ${ }^{39}$ They described a case of a dumbbell-shaped lesion with intradural and extradural components in the cervical spine along the C6 nerve root. The patient underwent 2 surgical procedures and received combination immunotherapy, including nivolumab (programmed cell death protein 1 [PD-1] death-ligand receptor), ipilimumab (cytotoxic T-lymphocyte antigen 4 [CTLA4]), and denosumab (receptor activator of nuclear factor kappa $b$ ligand antibodies), as these therapies have previously shown efficacy in treatment of primary melanoma. ${ }^{39}$ Unfortunately, this aggressive immunotherapy combination was ineffective, and the patient was subsequently readmitted with respiratory complications and died. ${ }^{39}$

Another report by Sahay et al. described 7 cases of melanotic spinal schwannoma occurring in the cranium or spinal cord. ${ }^{40}$ Four of these cases originated in the spine, in which 1 patient received immunotherapy following resection of a lumbar tumor. Following surgery and radiotherapy, the patient was treated with nivolumab, yet the tumor recurred after 1 year. This led to the addition of ipilimumab to the patient's regimen, although, in the following year, the patient's tumor burden worsened. Fortunately, at the 3-year follow-up, the patient was found to have stable disease after having received more than 46 cycles of nivolumab, a monoclonal antibody treatment typically used in melanoma management. ${ }^{40}$ While this outcome was favorable, the absence of larger-scale patient series or trials mandates more translational study to determine the true efficacy. This could include understanding the role of genomic profiling for hypermutation and prognosticating response, which to date has not been well studied in schwannomas. ${ }^{41}$

\section{Ependymoma}

Ependymomas are rare ectodermal tumors with multiple subtypes. ${ }^{42}$ Although the majority are benign in nature, they have the potential to metastasize through the spinal axis. ${ }^{42}$ In terms of adjuvant treatment so far, ependymomas have been demonstrated to be responsive to the vascular endothelial growth factor (VEGF) inhibitor, bevacizumab, as shown by Morris et al. ${ }^{42}$ Bevacizumab has previously shown efficacy in the management of vestibular schwannomas in the neurofibromatosis population..$^{42}$

Clinically, treating metastatic ependymomas with immunotherapy has demonstrated some degree of efficacy, as shown in a case report by Tapia Rico et al. ${ }^{8}$ The authors described the case of a patient with myxopapillary ependymoma who was subsequently treated with tislelizumab, an investigational monoclonal antibody targeted against anti-PD-1 following tumor resection. ${ }^{8}$ The disease course of the patient subsequently stabilized without major side effects at the 18-month follow-up. ${ }^{8}$ Similarly, Farschtschi et al. reported that patients with neurofibromatosis type 2 (NF2) with spinal ependymomas, who were subsequently treated with bevacizumab, demonstrated subjective clinical improvement. Furthermore, 5 of the 8 patients studied showed evidence of radiographic response defined by a $>$ $20 \%$ reduction in tumor volume at the 6- and 12-month follow-ups. ${ }^{43}$ Altogether, these results suggest that the use of immunotherapeutics may be beneficial in the setting of ependymoma tumorigenesis due to syndromic etiology.

\section{Secondary Spinal Tumors}

Multiple other tumor types can present as secondary growths along the spine, incorporating the spinal cord and surrounding vertebrae. Primary CNS lymphoma (PCNSL), multiple myeloma, metastatic tumors, and bone tumors all have a propensity to require more than surgical treatment. The use of immunotherapies is, therefore, highlighted below.

\section{Primary CNS Lymphoma}

PCNSL constitutes a group of unique and aggressive malignancies. These tumors are abnormal in that much of their invasive potential is limited to the CNS and its appendages; only rarely do PCNSLs extend beyond the CNS.44 Typically, PCNSLs arise from non-Hodgkin's lymphoma invading the brain, spine, meninges, and nerve roots. ${ }^{45}$ Spinal invasion is considered a relatively rare occurrence that, when present, may still have a significant impact on patients. For example, Williamson et al. documented a case of spinal PCNSL impinging on the conus medullaris and cauda equina.$^{46}$ Because of their location, spinal PCNSLs are more likely to present clinically before they are detectable on imaging, with patients typical- 
ly presenting with radiculopathy and other critical signs, such as sphincter dysfunction. ${ }^{47}$

Standard treatment of PCNSLs is multimodal, with well-established regimens including a combination of radiotherapy, chemotherapy, and immunotherapy ${ }^{43}$ For example, Wong et al. demonstrated the efficacy of an immunochemotherapeutic regimen involving combined use of temozolomide and the CD20 blocker rituximab in treatment of PCNSL. ${ }^{48}$ Rituximab has also been shown to be effective in conjunction with other therapeutic agents. Morris et al. demonstrated that patients who received rituximab in conjunction with methotrexate, procarbazine, and vincristine, followed by whole-brain radiotherapy and cytarabine, demonstrated a median overall survival of 6.6 years, with improved executive function and memory. ${ }^{42}$ Furthermore, Adhikari et al. reported on a pediatric patient with PCNSL who underwent treatment with rituximab chemoimmunotherapy followed by whole-brain radiotherapy. ${ }^{49}$ The patient received 5 cycles of rituximab, high-dose methotrexate, vincristine, and procarbazine. The patient was then treated with fractional whole-brain radiotherapy. At the 6-month follow-up, MRI showed complete radiological response. This report highlights the potential efficacy of immunotherapy combined with radiotherapy. While many of these studies investigated intracranial PCNSLs, these same regimens may have similar efficacy in spinal lesions.

\section{Multiple Myeloma}

Multiple myeloma may also metastasize to the vertebral column, resulting in pathological fracture. ${ }^{50}$ Indeed, the insidious nature of these metastases makes the resultant compression fractures the most common etiology of emergency spine surgery without a prior diagnosis of cancer. ${ }^{50}$ Immunotherapy has also shown promise in treatment of multiple myeloma, with monoclonal antibodies and CAR T-cell therapy targeting B-cell maturation antigen (BCMA) proving to be especially promising. ${ }^{51}$ For example, Raje et al. conducted a phase 1 study involving patients with either refractory or relapsed multiple myeloma who were treated with bb2121, a form of CAR T-cell therapy with an anti-BCMA single-chain variable fragment. ${ }^{52}$ Among their sample population, the objective response rate was $85 \%$, with $45 \%$ of patients demonstrating complete responses. Zhao et al. conducted a similar study with an anti-BCMA CAR T-cell therapeutic, LCARB38M, that targeted two distinct BCMA antigens. ${ }^{53}$ The overall response rate was $88 \%$, with 39 of the 57 patients achieving a complete response. These studies are promising; however, clinical studies with long-term follow-up that specifically investigate multiple myeloma in the spinal cord are necessary to determine the efficacy of immunotherapeutics for spinal cord tumors.

\section{Metastatic Tumors of the Spine}

Foremost among secondary spine tumors is metastatic melanoma, which has increased global incidence in recent years. ${ }^{54}$ Immunotherapy for metastatic melanoma can be largely clustered into 2 categories: cytokine-based therapies and checkpoint-control therapies. Cytokine therapy has largely focused on IL-2 and interferon- $\alpha$ (IFN- $\alpha$ ) as primary treatment modalities. ${ }^{55,56} \mathrm{IL}-2$ promotes growth and maturation of natural killer and $\mathrm{T}$ cells via the Ras/ MAPK and Jak/STAT pathways. ${ }^{57}$ IFN- $\alpha$ has demonstrated efficacy in melanoma treatment by promoting the development of natural killer and CD8+ T cells. When combined with chemotherapy, IFN- $\alpha$ has demonstrated a $60 \%$ response rate in patients with metastatic melanoma. ${ }^{57}$

One potential complication when considering cytokine immunotherapy is its demonstrated neurotoxicity, as previously cited ${ }^{42}$ However, other reports have suggested that this risk may not be absolute, particularly with respect to extramedullary spinal lesions, which impinge on, rather than directly involve, the spinal cord or vertebrae..$^{54,58}$

CAR T cells have also demonstrated efficacy in controlling metastatic ependymoma and medulloblastoma in murine xenograft models. ${ }^{17}$ Donovan et al. identified several novel medulloblastoma and ependymoma cell-surface targets that are not present on healthy tissue, and the authors were able to demonstrate significantly improved survival in mice infused with CAR T cells relative to wildtype mice. ${ }^{17}$

Immune checkpoint inhibitors may also offer a promising treatment modality for bony and intramedullary metastases, although most current data are a product of individual case reports. For example, Phillips et al. demonstrated regression of an intramedullary spinal metastasis following treatment with nivolumab. ${ }^{59}$ The utility of checkpoint inhibitors is also currently being investigated in clinical trials. For example, one group is currently recruiting patients for a clinical trial involving a combination therapy of the programmed death-ligand 1 (PD-L1) inhibitor, nivolumab, and stereotactic radiosurgery (registration no. NCT02716948, ClinicalTrials.gov).

\section{Bone Tumors}

Chondromas and chondrosarcomas are a class of bone tumors that may manifest in the spine. Typically, these tumors exist on opposite ends of a spectrum of disease, ranging from benign chondromas to potentially aggressive chondrosarcomas. ${ }^{60}$ Standard treatment involves bulk resection with or without radiotherapy, which has been shown to adequately control these lesions. ${ }^{60}$ However, recent scholarship has demonstrated a potential role for immunotherapy. Schwab et al. sought to identify overexpressed extracellular matrix proteins in spinal chondroma and chondrosarcoma. ${ }^{61}$ The authors identified multiple potential immunotherapy targets, including high-molecular-weight melanoma-associated antigen (HMW-MAA), which was found in $62 \%$ of chondromas and possesses a structure similar to that of a melanoma antigen. ${ }^{61}$

\section{Peripheral Nerve Tumors}

The rise in detection and treatment of peripheral nerve tumors signals an emerging interest in developing possible immunotherapies for these tumors. Although shadowed in development when compared with their spinal counterparts, peripheral nerve tumors, such as neurofibromas and malignant peripheral nerve tumors, may be vulnerable to specific immunotherapies. 
TABLE 1. Summary of clinical trials reviewed in the present study

\begin{tabular}{lccc}
\hline $\begin{array}{c}\text { Authors \& Year } \\
\text { (NCT no.) }\end{array}$ & $\begin{array}{c}\text { Immunotherapeutic } \\
\text { Agent }\end{array}$ & Pathology & Findings \\
\hline $\begin{array}{l}\text { Brown et al., 2018 } \\
\text { (NCT02208362) }\end{array}$ & CAR T cells & $\begin{array}{c}\text { Intracranial \& spinal } \\
\text { GBM }\end{array}$ & $\begin{array}{c}\text { Regression of all intracranial \& spinal tumors observed at 7.5 mos; increased } \\
\text { levels of cytokines \& immune cells in CSF }\end{array}$ \\
\hline $\begin{array}{l}\text { Rubenstein et al., 2013 } \\
\text { (NCT00098774) }\end{array}$ & Rituximab & $\begin{array}{c}\text { Intracranial \& spinal } \\
\text { PCNSL }\end{array}$ & $\begin{array}{c}\text { Rate of complete response was 66\%; overall 2-yr PFS was 0.57 at median } \\
\text { follow-up of 4.9 yrs }\end{array}$ \\
\hline $\begin{array}{l}\text { D'Adamo et al., 2019 } \\
\text { (NCT00837148) }\end{array}$ & Sorafenib & $\begin{array}{c}\text { Solid tumors, includ- } \\
\text { ing MPNSTs }\end{array}$ & $\begin{array}{c}\text { Median PFS of 13.4 wks; disease control rate of 46\% } \\
\text { Jakacki et al., 2017 }\end{array}$ \\
$\begin{array}{l}\text { (NCT00021541) } \\
\text { (N8 }\end{array}$ & $\begin{array}{c}\text { Pegylated } \\
\text { interferon }\end{array}$ & $\begin{array}{c}\text { NF2-related periph- } \\
\text { eral nerve tumors }\end{array}$ & $\begin{array}{c}\text { Imaging response observed in 4 pts; clinical response observed in 3 pts; me- } \\
\text { dian TTP was 29.4 mos in treatment group vs 11.8 mos in placebo group }\end{array}$ \\
\hline
\end{tabular}

GBM = glioblastoma; $\mathrm{NCT}$ = National Clinical Trial; $\mathrm{PFS}=$ progression-free survival; pts = patients; $\mathrm{TTP}=$ time to progression .

\section{Neurofibromas}

Neurofibromas have also come under consideration for immunotherapeutic management, particularly in the setting of neurofibromatosis type 1 (NF1) and NF2. This stems from previous reports demonstrating dysregulation in the immune response of patients with neurofibromatosis that may result in the formation of neurofibromas. ${ }^{62}$ For example, the macrophages of patients with neurofibromatosis demonstrate markedly different expression of inflammatory genes relative to those of healthy patients, and neurofibromas have been previously associated with interferon dysregulation. ${ }^{62}$ However, despite these advances, immunotherapeutics targeting these altered immune responses have not yet been demonstrated in the literature.

\section{Malignant Peripheral Nerve Sheath Tumors}

Immunotherapy for management of malignant peripheral nerve sheath tumors (MPNSTs) is largely limited to in vitro and in vivo investigations, although some small-scale clinical trials have been reported. Multiple studies have investigated the utility of oncolytic viruses in xenograft models of MPNSTs. ${ }^{11,63}$ The immunotherapeutic utility of oncolytic viruses lies in their ability to generate host immune responses following replication of viral vectors and expression of neurovirulent proteins on the tumor cell surface. ${ }^{11}$ Furthermore, a recent systematic review demonstrated that oncolytic herpesviruses were moderately to highly effective in limiting MPNST cellline growth. ${ }^{64}$ This was demonstrated by Antoszczyk et al. in a preclinical study in which mouse NF1 MPNST cell lines and human NF1 MPNST stemlike cells were implanted into the sciatic nerve of murine models. ${ }^{65}$ Oncolytic herpes simplex viruses were injected intratumorally, resulting in tumor growth inhibition and improved overall survival. Similarly, Currier et al. demonstrated that a combination of a small molecule Aurora A kinase inhibitor and HSV1716 reduced tumor growth and also increased survival in xenograft models with MPNST and neuroblastomas. The results of these preclinical studies have indicated the promising potential of oncolytic herpes simplex viruses in treating MPNSTs. ${ }^{63}$

Several immunomodulatory techniques centered on macrophages are also currently under investigation for treatment in MPNSTs and other neurofibroma-related pe- ripheral nerve tumors. Previous studies have established that macrophages play a critical role in the growth of MPNSTs and other NF1-associated tumors. ${ }^{66}$ However, direct inhibition of the FMS kinase pathway, which is critical for macrophage function, only resulted in decreased tumor cell growth in already established tumors, while inhibition in unestablished tumors paradoxically resulted in increased tumor cell growth. ${ }^{12}$ Thus, current cellular immunomodulatory therapies are poorly understood and require further research prior to development of potential therapeutics.

\section{Clinical Trials}

There are 2 clinical trials reported in the literature that discuss the use of immunotherapy in treating spine and peripheral nerve tumors (Table 1). ${ }^{3,26,67-69}$ All results appear promising, but greater cohort validation is needed.

\section{Spine Tumors}

Brown et al. demonstrated the efficacy of CAR T-cell therapy in the management of a patient who had multifocal glioblastoma with both a primary brain lesion and spine metastases (registration no. NCT02208362, ClinicalTrials.gov). ${ }^{13}$ Following the first 3 intraventricular infusions of CAR T cells, the patient demonstrated a marked reduction in both cerebral and spinal tumors. By the fifth infusion, the patient demonstrated $77 \%$ to $100 \%$ reduction in these lesions. ${ }^{13}$ At the 7.5-month follow-up, the patient did not demonstrate recurrence of any lesions. The authors also monitored the inflammatory response following CAR T-cell infusion and demonstrated a significant increase in CSF cytokines following CAR T-cell infusion..$^{13}$

Another study by Rubenstein et al. reported a multicenter clinical trial exploring the use of chemotherapy and immunotherapy in patients with PCNSL in the brain and spine (registration no. NCT00098774, ClinicalTrials. gov). ${ }^{69}$ In this study, 44 patients were treated with methotrexate, temozolomide, and rituximab. The rate of complete response to therapy was $66 \%$ and the overall 2-year progression-free survival was 0.57 with a median followup of 4.9 years. This result is comparable to that of patients with PCNSL who underwent treatment with whole-brain radiation. While the results of this study were favorable, the efficacy of the immunotherapeutic agent studied was 
confounded by the addition of high-dose chemotherapy and may require further investigation.

\section{Peripheral Nerve Tumors}

D'Adamo et al. investigated the use of the small molecular antiangiogenic oral agent sorafenib (targeting VEGF receptor, platelet-derived growth factor receptor [PDGFR], and RAF kinases) and the cytotoxin dacarbazine administered as a combination immunotherapy in 37 patients with various solid tumors, including 4 with MPNSTs (registration no. NCT00837148, ClinicalTrials.gov). ${ }^{67}$ Their results showed that, across tumor subtypes, patients who received sorafenib and dacarbazine therapy had a median progression-free survival of 13.4 weeks ${ }^{67}$ Since sorafenib has previously been associated with disease stabilization rather than clinical response, the authors reported the response to combination therapy as the disease control rate, which was demonstrated to be $46 \%$ across all tumor subtypes. ${ }^{67} \mathrm{How}-$ ever, the study was marked by several concerns regarding hematological toxicities inherent to dacarbazine treatment. Of the first 25 patients, 15 required a dose reduction of dacarbazine. ${ }^{67}$

Clinical trials have also investigated the utility of intravenous pegylated interferon in management of unresectable and progressive NF2-related peripheral nerve tumors. ${ }^{38,68}$ However, the precise use of pegylated interferon in the management of these lesions is unclear. For example, Jakacki et al. reported a $20 \%$ reduction in tumor load at the follow-up ( $\geq 4$ weeks after administration) in a modest $4.9 \%$ of patients (registration no. NCT00021541, ClinicalTrials.gov) ${ }^{68}$ However, all patients in the study by Kebudi et al. demonstrated improved pain relief. ${ }^{38}$ Thus, there may be a role for pegylated interferon with respect to pain control or other secondary outcomes, but its use as a primary means of tumor reduction may be limited.

\section{Conclusions}

Immunotherapy for spinal and peripheral neve tumors is an active area of research in neuro-oncology. While preclinical and clinical studies have shown some efficacy, additional larger-scale randomized controlled trials are needed before substantial conclusions may be drawn from the current state of the literature. Future clinical studies for translation will focus on the optimal therapy type and administration route to best target these tumors, which often preclude total resection given their proximity to the neural elements of the spine.

\section{References}

1. Chamberlain MC, Tredway TL. Adult primary intradural spinal cord tumors: a review. Curr Neurol Neurosci Rep. 2011; 11(3):320-328.

2. Hu J, Liu T, Han B, Tan S, Guo H, Xin Y. Immunotherapy: a potential approach for high-grade spinal cord astrocytomas. Front Immunol. 2021;11:582828.

3. Belakhoua SM, Rodriguez FJ. Diagnostic pathology of tumors of peripheral nerve. Neurosurgery. 2021;88(3):443-456.

4. Agliardi G, Liuzzi AR, Hotblack A, et al. Intratumoral IL-12 delivery empowers CAR-T cell immunotherapy in a preclinical model of glioblastoma. Nat Commun. 2021;12(1):444.

5. He G, Liu X, Pan X, Ma Y, Liu X. Cytotoxic T lymphocyte antigen-4 (CTLA-4) expression in chordoma and tumorinfiltrating lymphocytes (TILs) predicts prognosis of spinal chordoma. Clin Transl Oncol. 2020;22(12):2324-2332.

6. Majd N, Dasgupta P, de Groot J. Immunotherapy for neurooncology. Adv Exp Med Biol. 2020;1244:183-203.

7. Shankar GM, Choi BD, Grannan BL, Oh K, Shin JH. Effect of immunotherapy status on outcomes in patients with metastatic melanoma to the spine. Spine (Phila Pa 1976). 2017; 42(12):E721-E725.

8. Tapia Rico G, Townsend A, Price T, Patterson K. Metastatic myxopapillary ependymoma treated with immunotherapy achieving durable response. BMJ Case Rep. 2020;13(12): e236242.

9. Wang SS, Bandopadhayay P, Jenkins MR. Towards immunotherapy for pediatric brain tumors. Trends Immunol. 2019; 40(8):748-761.

10. Ye L, Park JJ, Dong MB, et al. In vivo CRISPR screening in CD8 T cells with AAV-Sleeping Beauty hybrid vectors identifies membrane targets for improving immunotherapy for glioblastoma. Nat Biotechnol. 2019;37(11):1302-1313.

11. Maldonado AR, Klanke C, Jegga AG, et al. Molecular engineering and validation of an oncolytic herpes simplex virus type 1 transcriptionally targeted to midkine-positive tumors. J Gene Med. 2010;12(7):613-623.

12. O'Rourke DM, Nasrallah MP, Desai A, et al. A single dose of peripherally infused EGFRvIII-directed CAR T cells mediates antigen loss and induces adaptive resistance in patients with recurrent glioblastoma. Sci Transl Med. 2017;9(399): eaaa0984.

13. Brown CE, Aguilar B, Starr R, et al. Optimization of IL13R $\alpha 2$-targeted chimeric antigen receptor T cells for improved anti-tumor efficacy against glioblastoma. Mol Ther. 2018;26(1):31-44.

14. Brown CE, Alizadeh D, Starr R, et al. Regression of glioblastoma after chimeric antigen receptor T-cell therapy. $N$ Engl J Med. 2016;375(26):2561-2569.

15. Desjardins A, Gromeier M, Herndon JE II, et al. Recurrent glioblastoma treated with recombinant poliovirus. $N$ Engl $J$ Med. 2018;379(2):150-161.

16. Sridhar P, Petrocca F. Regional delivery of chimeric antigen receptor (CAR) T-cells for cancer therapy. Cancers (Basel). 2017;9(7):E92.

17. Donovan LK, Delaidelli A, Joseph SK, et al. Locoregional delivery of CAR T cells to the cerebrospinal fluid for treatment of metastatic medulloblastoma and ependymoma. Nat Med. 2020;26(5):720-731.

18. Huppert LA, Melisko ME, Glastonbury CM, Khanafshar E, Daud AI. Treatment of metastatic melanoma with leptomeningeal disease using intrathecal immunotherapy. JCO Oncol Pract. 2020;16(11):757-759.

19. Raj VS, Lofton L. Rehabilitation and treatment of spinal cord tumors. J Spinal Cord Med. 2013;36(1):4-11.

20. Hsu S, Quattrone M, Ostrom Q, Ryken TC, Sloan AE, Barnholtz-Sloan JS. Incidence patterns for primary malignant spinal cord gliomas: a Surveillance, Epidemiology, and End Results study. J Neurosurg Spine. 2011;14(6):742-747.

21. Bagley SJ, Desai AS, Linette GP, June CH, O'Rourke DM. CAR T-cell therapy for glioblastoma: recent clinical advances and future challenges. Neuro Oncol. 2018;20(11): 1429-1438.

22. Cui J, Zhang Q, Song Q, et al. Targeting hypoxia downstream signaling protein, CAIX, for CAR T-cell therapy against glioblastoma. Neuro Oncol. 2019;21(11):1436-1446.

23. Prinzing BL, Gottschalk SM, Krenciute G. CAR T-cell therapy for glioblastoma: ready for the next round of clinical testing? Expert Rev Anticancer Ther. 2018;18(5):451-461.

24. Raco A, Esposito V, Lenzi J, Piccirilli M, Delfini R, Cantore G. Long-term follow-up of intramedullary spinal cord tumors: a series of 202 cases. Neurosurgery. 2005;56(5):972-981. 
25. Tobin MK, Geraghty JR, Engelhard HH, Linninger AA, Mehta AI. Intramedullary spinal cord tumors: a review of current and future treatment strategies. Neurosurg Focus. 2015;39(2):E14.

26. Ahmed N, Brawley V, Hegde M, et al. HER2-specific chimeric antigen receptor-modified virus-specific $\mathrm{T}$ cells for progressive glioblastoma: a phase 1 dose-escalation trial. JAMA Oncol. 2017;3(8):1094-1101.

27. Chow KK, Naik S, Kakarla S, et al. T cells redirected to EphA2 for the immunotherapy of glioblastoma. Mol Ther. 2013;21(3):629-637.

28. Ferluga S, Debinski W. Ephs and ephrins in malignant gliomas. Growth Factors. 2014;32(6):190-201.

29. Sampson JH, Archer GE, Mitchell DA, Heimberger AB, Bigner DD. Tumor-specific immunotherapy targeting the EGFRvIII mutation in patients with malignant glioma. Semin Immunol. 2008;20(5):267-275.

30. Sengupta S, Thaci B, Crawford AC, Sampath P. Interleukin-13 receptor alpha 2-targeted glioblastoma immunotherapy. Biomed Res Int. 2014;2014:952128.

31. Mount CW, Majzner RG, Sundaresh S, et al. Potent antitumor efficacy of anti-GD2 CAR T cells in H3-K27M+ diffuse midline gliomas. Nat Med. 2018;24(5):572-579.

32. Coxon AT, Johanns TM, Dunn GP. An innovative immunotherapy vaccine with combination checkpoint blockade as a first line treatment for glioblastoma in the context of current treatments. Mo Med. 2020;117(1):45-49.

33. Kong Z, Wang Y, Ma W. Vaccination in the immunotherapy of glioblastoma. Hum Vaccin Immunother. 2018;14(2):255-268.

34. Medikonda R, Dunn G, Rahman M, Fecci P, Lim M. A review of glioblastoma immunotherapy. J Neurooncol. 2021; 151(1):41-53.

35. Chheda ZS, Kohanbash G, Okada K, et al. Novel and shared neoantigen derived from histone 3 variant H3.3K 27M mutation for glioma T cell therapy. J Exp Med. 2018;215(1): 141-157.

36. Ochs K, Ott M, Bunse T, et al. K27M-mutant histone-3 as a novel target for glioma immunotherapy. OncoImmunology. 2017;6(7):e1328340.

37. Wu L, Yao N, Chen D, Deng X, Xu Y. Preoperative diagnosis of intramedullary spinal schwannomas. Neurol Med Chir (Tokyo). 2011;51(9):630-634.

38. Kebudi R, Cakir FB, Gorgun O. Interferon- $\alpha$ for unresectable progressive and symptomatic plexiform neurofibromas. $J$ Pediatr Hematol Oncol. 2013;35(3):e115-e117.

39. Solomou G, Dulanka Silva AH, Wong A, Pohl U, Tzerakis N. Extramedullary malignant melanotic schwannoma of the spine: case report and an up to date systematic review of the literature. Ann Med Surg (Lond). 2020;59:217-223.

40. Sahay A, Epari S, Gupta P, et al. Melanotic schwannoma, a deceptive misnomer for a tumor with relative aggressive behavior: a series of 7 cranial and spinal cases. Int J Surg Pathol. 2020;28(8):850-858.

41. Sinnadurai M, McDonald KL. Immune checkpoint inhibition and its relationship with hypermutation phenoytype as a potential treatment for glioblastoma. J Neurooncol. 2017; 132(3):359-372.

42. Morris KA, Afridi SK, Evans DG, et al. The response of spinal cord ependymomas to bevacizumab in patients with neurofibromatosis Type 2. J Neurosurg Spine. 2017;26(4): 474-482.

43. Farschtschi S, Merker VL, Wolf D, et al. Bevacizumab treatment for symptomatic spinal ependymomas in neurofibromatosis type 2. Acta Neurol Scand. 2016;133(6):475-480.

44. Ferreri AJM, Holdhoff M, Nayak L, Rubenstein JL. Evolving treatments for primary central nervous system lymphoma. Am Soc Clin Oncol Educ Book. 2019;39:454-466.

45. Hochberg FH, Baehring JM, Hochberg EP. Primary CNS lymphoma. Nat Clin Pract Neurol. 2007;3(1):24-35.
46. Williamson TJ, Wang M, Clark J, Williams J, Drnda A. Primary intradural Hodgkin lymphoma of the conus medullaris and cauda equina: case report. CNS Oncol. 2020;9(3):CNS52.

47. Herrlinger U, Weller M, Küker W. Primary CNS lymphoma in the spinal cord: clinical manifestations may precede MRI detectability. Neuroradiology. 2002;44(3):239-244.

48. Wong ET, Tishler R, Barron L, Wu JK. Immunochemotherapy with rituximab and temozolomide for central nervous system lymphomas. Cancer. 2004;101(1):139-145.

49. Adhikari N, Biswas A, Bakhshi S, Khanna G, Suri V. A rare case of paediatric primary central nervous system lymphoma treated with high-dose methotrexate and rituximab-based chemoimmunotherapy and whole brain radiotherapy followed by tumour bed boost with three-dimensional conformal radiation technique. Childs Nerv Syst. 2018;34(9):1777-1783.

50. Burks JD, Elarjani T, Jamshidi AM, Govindarajan V, Levi AD. Vertebral multiple myeloma with pathological fracture: the most common etiology for emergency spine surgery in patients with no cancer diagnosis on admission. Neurosurg Focus. 2021;50(5):E2.

51. Rasche L, Hudecek M, Einsele $H$. What is the future of immunotherapy in multiple myeloma? Blood. 2020;136(22): 2491-2497.

52. Raje N, Berdeja J, Lin Y, et al. Anti-BCMA CAR T-cell therapy bb2121 in relapsed or refractory multiple myeloma. $N$ Engl J Med. 2019;380(18):1726-1737.

53. Zhao WH, Liu J, Wang BY, et al. A phase 1, open-label study of LCAR-B38M, a chimeric antigen receptor T cell therapy directed against B cell maturation antigen, in patients with relapsed or refractory multiple myeloma. J Hematol Oncol. 2018;11(1):141.

54. Russell-Jones R. Interferon-alpha therapy for melanoma. Clin Exp Dermatol. 2000;25(1):1-6.

55. Chu MB, Fesler MJ, Armbrecht ES, Fosko SW, Hsueh E, Richart JM. High-dose interleukin-2 (HD IL-2) therapy should be considered for treatment of patients with melanoma brain metastases. Chemother Res Pract. 2013;2013:726925.

56. Legha SS. Durable complete responses in metastatic melanoma treated with interleukin-2 in combination with interferon alpha and chemotherapy. Semin Oncol. 1997;24(1)(suppl 4): S39-S43.

57. Hodi FS, Oble DA, Drappatz J, et al. CTLA-4 blockade with ipilimumab induces significant clinical benefit in a female with melanoma metastases to the CNS. Nat Clin Pract Oncol. 2008;5(9):557-561.

58. Grimm S, Chamberlain MC. Adult primary spinal cord tumors. Expert Rev Neurother. 2009;9(10):1487-1495.

59. Phillips KA, Gaughan E, Gru A, Schiff D. Regression of an intramedullary spinal cord metastasis with a checkpoint inhibitor: a case report. CNS Oncol. 2017;6(4):275-280.

60. McLoughlin GS, Sciubba DM, Wolinsky JP. Chondroma/ chondrosarcoma of the spine. Neurosurg Clin N Am. 2008; 19(1):57-63.

61. Schwab JH, Boland PJ, Agaram NP, et al. Chordoma and chondrosarcoma gene profile: implications for immunotherapy. Cancer Immunol Immunother. 2009;58(3):339-349.

62. Walker JA, Upadhyaya M. Emerging therapeutic targets for neurofibromatosis type 1. Expert Opin Ther Targets. 2018; 22(5):419-437.

63. Currier MA, Sprague L, Rizvi TA, et al. Aurora A kinase inhibition enhances oncolytic herpes virotherapy through cytotoxic synergy and innate cellular immune modulation. Oncotarget. 2017;8(11):17412-17427.

64. Martin E, Lamba N, Flucke UE, et al. Non-cytotoxic systemic treatment in malignant peripheral nerve sheath tumors (MPNST): a systematic review from bench to bedside. Crit Rev Oncol Hematol. 2019;138:223-232.

65. Antoszczyk S, Spyra M, Mautner VF, et al. Treatment of orthotopic malignant peripheral nerve sheath tumors with 
oncolytic herpes simplex virus. Neuro Oncol. 2014;16(8): 1057-1066.

66. Prada CE, Jousma E, Rizvi TA, et al. Neurofibroma-associated macrophages play roles in tumor growth and response to pharmacological inhibition. Acta Neuropathol. 2013;125(1): $159-168$.

67. D'Adamo DR, Dickson MA, Keohan ML, et al. A phase II trial of sorafenib and dacarbazine for leiomyosarcoma, synovial sarcoma, and malignant peripheral nerve sheath tumors. Oncologist. 2019;24(6):857-863.

68. Jakacki RI, Dombi E, Steinberg SM, et al. Phase II trial of pegylated interferon alfa- $2 b$ in young patients with neurofibromatosis type 1 and unresectable plexiform neurofibromas. Neuro Oncol. 2017;19(2):289-297.

69. Rubenstein JL, Hsi ED, Johnson JL, et al. Intensive chemotherapy and immunotherapy in patients with newly diagnosed primary CNS lymphoma: CALGB 50202 (Alliance 50202). J Clin Oncol. 2013;31(25):3061-3068.

\section{Disclosures}

Dr. Levi: consultant for Medtronic and grants from the Department of Defense and NIH.

\section{Author Contributions}

Conception and design: all authors. Acquisition of data: Bryant, Lu, Govindarajan, Perez-Roman. Analysis and interpretation of data: all authors. Drafting the article: all authors. Critically revising the article: all authors. Reviewed submitted version of manuscript: Levi, Bryant, Govindarajan, Perez-Roman. Approved the final version of the manuscript on behalf of all authors: Levi.

\section{Correspondence}

Allan D. Levi: Lois Pope LIFE Center, Miami, FL. alevi@med. miami.edu. 